\title{
Prevalência de sedentarismo em adultos obesos e sobrepesados
}

\section{Prevalence of sedentarism in obese and overweight adults}

\author{
Carlos Alexandre Molena Fernandes' $\bullet$ Maria Luiza Costa Borim ${ }^{2} \bullet$ Maria Antonia Ramos Costa ${ }^{3}$ \\ Heloá Costa Borim Christinelli ${ }^{4} \bullet$ Kelly Paviani Stevanato ${ }^{5} \bullet$ Julie Tayná Nagai Henrique $^{6}$
}

\begin{abstract}
RESUMO
Objetivo:Verificar a prevalência de sedentarismo em adultos com sobrepeso e obesidade e os fatores associados. Metodologia: Estudo piloto, descritivo transversal com abordagem quantitativa. Realizado com adultos, de ambos os sexos e idade entre 18 e 50 anos, residentes em um município no noroeste do Paraná. A amostra por conveniência contou com 50 participantes que foram submetidos a avaliação de medidas antropométricas de peso, estatura e circunferência de cintura para determinar quais atendiam ao critério de inclusão: estar com sobrepeso ou obesidade. Os 50 indivíduos foram classificados conforme o resultado do cálculo do IMC, e 34 atenderam aos critérios de inclusão sendo convidados a responder o Questionário Internacional de Atividade Física (IPAQ). Resultados: Este estudo apresentou prevalência de sedentarismo em $58,8 \%$ dos adultos sobrepesados e obesos, identificou como comportamentos sedentários mais comuns nesta população o uso de computadores e televisores, e demonstrou correlação positiva entre o sexo feminino e o sedentarismo neste público-alvo. Conclusão:A maioria dos participantes apresentou comportamento sedentário, sendo mais frequente entre as mulheres.
\end{abstract}

Palavras-chave: Estilo deVida Sedentário;Atividade Física; Obesidade.

\begin{abstract}
Objective:To verify the prevalence of physical inactivity in overweight and obese adults and the associated factors. Methodology: Pilot study, cross-sectional with a quantitative approach. Performed with adults, of both sexes and aged between 18 and 50 years, living in a municipality in the northwest of Paraná. The convenience sample included 50 participants who underwent anthropometric measurements of weight, height and waist circumference to determine which met the inclusion criteria: being overweight or obese. The 50 individuals were classified according to the result of the BMI calculation, and 34 met the inclusion criteria and were invited to answer the International Physical Activity Questionnaire (IPAQ). Results: This study showed a prevalence of sedentary lifestyle in $58.8 \%$ of overweight and obese adults, identified as the most common sedentary behaviors in this population the use of computers and televisions, and demonstrated a positive correlation between females and sedentary lifestyle in this target audience. Conclusion: Most participants showed sedentary behavior, being more frequent among women.

Keywords: Sedentary Lifestyle; Physical activity; Obesity.
\end{abstract}

I Diretor de Pós-Graduação da Universidade Estadual do Paraná - UNESPAR. Possui graduação em Educação Física pela Universidade Estadual de Maringá (2003), Especialização em Promoção à Saúde do Homem (2004), Mestrado em Ciências da Saúde pela Universidade Estadual de Maringá (2006) e Doutorado em Ciências Farmacêuticas pela Universidade Estadual de Maringá (2010). Docente Adjunto C da UNESPAR (Universidade Estadual do Paraná - Campus de Paranavai). Atualmente é professor permanente no programa de Pós-Graduação Stricto Sensu (Mestrado e Doutorado em Enfermagem) da Universidade Estadual de Maringá - UEM.

2 Graduação em Educação Física pela Universidade Estadual de Maringá

3 Graduada em Enfermagem e Obstetrícia, Mestre pela Universidade Estadual Paulista - Júlio de Mesquita Filho, Pró-Reitora de Pesquisa e Pós-Graduação, Coordenadora do Comitê de Ética em Pesquisa com Seres Humanos.

4 Graduada em enfermagem, mestre em tecnologia da saúde pela Pontifícia Universidade Católica do Paraná, doutora da em enfermagem pela Universidade Estadual de Maringá. Professora do curso de Enfermagem da Universidade Estadual do Paraná.

5 Graduação em Enfermagem pela Faculdade Estadual de Educação, Ciências e Letras de Paranavaí (2008). Especialista em Cuidados Intensivos em UTI pelo Instituto Brasileiro de Estudos e Pesquisas Socioeconômicas - INBRAPE - Londrina - Pr. (2009) e Especialista em Análise de Situação de Saúde pela Universidade Federal de Goiás - UFG - GO (2016). Mestrado em Ciências da Saúde pela Universidade Estadual de Maringá - UEM (2019). Docente colaboradora na Universidade Estadual do Paraná - UNESPAR.

6 Acadêmica do curso de enfermagem. Universidade Estadual do Paraná- UNESPAR Campus Paranavai 


\section{INTRODUÇÃO}

Atualmente duas definições de sedentarismo têm sido discutidas, uma relacionada ao excesso de comportamentos sedentários e outra relacionada a prática escassa de atividade física (AF). Contudo, ambos os estudos direcionados ao comportamento sedentário acabam por revelar os malefícios que o mesmo traz para o organismo'.

Considera-se comportamento sedentário qualquer comportamento realizado no período de vigília, caracterizado por um gasto energético $\leq 1,5$ equivalentes metabólicos (METs), na posição sentada, reclinada ou deitada para indivíduos acima de 18 anos de idade. Exemplifica-se este comportamento por meio do hábito de utilizar equipamentos eletrônicos como televisão, computador, telefone e tablet, nas posições sentada, deitada ou reclinada ${ }^{2}$.

Para se alcançar níveis de AF considerados satisfatórios, de acordo com as recomendações internacionais, os adultos acima de 18 anos devem cumprir 150 minutos de AF de intensidade vigorosa (dança, jardinagem, tarefas domésticas) à moderada, como corrida, ciclismo e natação durante a semana ou o mínimo de 75 minutos de atividade vigorosa por semana ${ }^{2,3}$.

O sedentarismo foi classificado como o quarto maior fator de risco de mortalidade global, deste modo, o mesmo acaba sendo um dos fatores de risco mais encontrados na população para o surgimento de doenças cardiovasculares (DCV) e doenças crônico-degenerativas tais como diabetes mellitus tipo II, hipertensão arterial sistêmica (HAS), síndrome metabólica e dislipidemia, levando ao crescimento da morbimortalidade cardiovascular, constatando riscos para patologias cardíacas e cerebrais ${ }^{4}$.

Em relação à insuficiência de $A F$, um estudo realizado com II.293 participantes, demonstrou que, destes, $14,4 \%(n=1.635)$ desenvolveram HAS, a qual esteve associada à insuficiência de AF dos entrevistados juntamente a outros fatores de risco como tabagismo e alimentação ${ }^{5}$.

Em todo o mundo, I em cada 4 adultos não tem o hábito de praticar AF suficientemente ${ }^{6}$. No Brasil, dentre 53.210 indivíduos com mais de 18 anos no ano de 2016 , apenas $37,6 \%$ praticava algum tipo de $\mathrm{AF}^{7}$. Este hábito inadequado está entre os principais fatores de risco para o surgimento de doenças crônicas não transmissíveis (DCNT), além de outros fatores como tabagismo, alimentação não saudável e alcoolismo.Vale ressaltar que tratam-se de fatores de risco modificáveis, deste modo, podem sofrer interferência direta por meio da mudança destes hábitos danosos à saúde, para que haja uma meIhoria na qualidade de vida do indivíduo ${ }^{8}$.

Diante do exposto, questiona-se: Qual a prevalência de sedentarismo entre indivíduos com excesso de peso? Neste sentido, o objetivo deste estudo foi verificar a prevalência de sedentarismo em adultos com sobrepeso e obesidade e os fatores associados.

\section{METODOLOGIA}

Trata-se de um estudo piloto de caráter descritivo transversal com abordagem quantitativa. A coleta de dados foi realizada com adultos, de ambos os sexos, com idade entre 18 e 50 anos, residentes no município do estudo no noroeste do Paraná, pertencentes a uma Unidade Básica de Saúde (UBS). A amostra se constituiu por conveniência, por se tratar de um estudo piloto. Deste modo, todos os indivíduos que adentraram na UBS onde o estudo foi realizado nos dias 29/04/201 9 e 30/04/2019 foram convidados a participar da pesquisa.

Nos dois dias de coleta de dados, 50 indivíduos que adentraram à UBS de estudo aceitaram passar pela avaliação de medidas antropométricas de peso, estatura e circunferência de cintura. Essa avaliação de pré-inclusão foi necessária para determinar quais indivíduos atendiam aos critérios de inclusão que eram: estar com sobrepeso ou obesidade, a partir de pontos de corte do Índice de Massa Corporal (IMC) $\geq 25^{9}$ e circunferência de cintura $\geq 88 \mathrm{~cm}$ para mulheres ou $\geq 102 \mathrm{~cm}$ para homens ${ }^{10}$.

Para a verificação de peso, os indivíduos foram orientados a permanecerem em posição ortostática, posicionados sobre o centro da balança digital antropométrica, com os pés descalços, trajando roupas leves. Para medida da estatura foi utilizada uma régua antropométrica, e os mesmos foram orientados a permanecerem eretos e na posição ortostática, com os pés descalços e unidos. A avaliação do IMC foi realizada pela divisão do peso corporal (em quilograma) pelo quadrado da altura (em metro).A classificação do IMC foi definida em obesidade, sobrepeso, peso normal e desnutrição de acordo com a Organização Mundial da Saúde (OMS) ${ }^{9}$.

Para a medida da circunferência de cintura os indivíduos foram orientados a deixar a região da cintura livre de roupas e a permanecer em pé, descalço, com a blusa levantada, os braços flexionados e cruzados em frente ao tórax, pés afastados, abdômen relaxado e respirando normalmente. Foi utilizada uma fita métrica não flexível diretamente sobre a pele na região entre a última costela e a crista ilíaca, sendo a leitura feita no momento da expiração".

Posteriormente a essa avaliação antropométrica, os 50 indivíduos foram classificados conforme o resultado do cálculo do IMC, e a amostra do estudo foi composta por 34 participantes que atenderam aos critérios de inclusão sendo convidados então, a responder o Questionário Internacional de Atividade Física (IPAQ) ${ }^{12}$, aplicado pela pesquisadora, com vistas a definir o perfil desta população, assim como seus hábitos e estilo de vida relacionados à prática de atividade física.

Os dados coletados foram organizados em planilha da ferramenta Excel e analisados, por meio de estatística descritiva, frequência simples e regressão linear utilizan- 
do-se o software Epi info 7, considerando nível de confiança de $95 \%$, erro amostral de $5 \%$ e $p$-valor $\leq 0,5$.

Para a análise de correlação entre as variáveis foi utilizado a regressão linear, sendo assim as variáveis com mais de uma categoria foram agrupadas da seguinte forma: idade 18 a 34 ou 35 a 50; raça e cor foram categorizados como branco ou não branco (pardos, amarelos e indígenas); escolaridade até 8 anos de estudo e 8 anos ou mais de estudo; renda familiar até 3 salários mínimos ou mais de 3 salários mínimos; tabagismo nunca fumou (não) ou fumante/ex-fumante (sim); consumo de bebida alcoólica nunca consumiu (não) ou socialmente/regularmente/ quase sempre/todos os dias (sim); as variáveis consumo de frutas, legumes e verdura, carne vermelha, carne branca, doces, gorduras foram agrupadas da seguinte maneira: nunca ( 0$)$, as vezes ( 1$)$, regularmente/quase sempre/todos os dias (2).

Para a classificação da AF os indivíduos foram classificados como muito ativos, ativos, irregularmente ativos ou sedentários ${ }^{12}$. Para a regressão linear foram agrupados em ativos fisicamente (muito ativos e ativos) e insuficientemente ativos (irregularmente ativos e sedentários). Considerando as duas definições de sedentarismo apresentadas anteriormente, uma relacionada ao excesso de comportamentos sedentários e outra relacionada a prática escassa de $\mathrm{AF}^{\prime}$, este estudo considerou como sedentários, todos os indivíduos classificados como irregularmente ativos ou sedentários de acordo com o questionário utilizado.

Esta pesquisa é um estudo piloto do projeto intitulado: "Eficácia de um programa multiprofissional na avaliação de fatores de risco cardiometabólico e tratamento da obesidade abdominal em dois municípios do noroeste do Paraná”, liderado pela Universidade Estadual de Maringá em parceria com a Universidade Estadual do Paraná - Campus Paranavaí, financiado pela Fundação Araucária, com aprovação do comitê de ética em pesquisa da Universidade Estadual de Maringá, sob CEP protocolo $n^{\circ}$ 2.655.268. Todos os participantes do estudo foram orientados sobre o mesmo, e consentiram sua participação por meio da assinatura do Termo de Consentimento Livre e Esclarecido (TCLE).

\section{RESULTADOS}

Participaram do estudo 34 indivíduos com a classificação de IMC como sobrepesados ( $n=17) 50 \%$ e obesos $(n=17) 50 \%$. A maioria do sexo feminino $(n=23) 68 \%$, não-brancos ( $n=19) 55 \%$, com mais de 8 anos de estudo $(n=22) 65 \%$, trabalhavam $(n=23) 68 \%$ e tinham renda familiar menor ou igual a 3 salários mínimos $(n=29) 85 \%$. A média de idade apresentada foi de 34,76 anos (mín. 19 e máx. 49). As características sociodemográficas dos participantes é apresentada na Tabela I.
A Tabela 2 apresenta a distribuição dos indivíduos realizada a partir da classificação do IMC e da classificação do nível de AF.

Os comportamentos sedentários informados pelos participantes são apresentados na Tabela 3.

Ao analisar a relação entre o uso de drogas lícitas e o perfil de atividade física dos participantes foi observado que, dentre os $41 \%$ que relataram consumir bebida alcoólica, $14 \%$ foram classificados como muito ativos fisicamente, $21 \%$ como ativos fisicamente, $50 \%$ como irregularmente ativos e $14 \%$ como sedentários. Por sua vez, dos 5 indivíduos tabagistas, $80 \%$ são irregularmente ativos e $20 \%$ são sedentários.

A Tabela 4 apresenta o perfil de consumo alimentar relatado pelos participantes de acordo com o nível de AF.

Durante a aplicação do questionário, os participantes responderam se consideravam a sua alimentação geral como saudável ou não, sendo que, 18 afirmaram que não, destes, a maioria eram insuficientemente ativos fisicamente ( 8 irregularmente ativos e 3 sedentários).

Dentre as variáveis analisadas, ser do sexo feminino apresentou relação estatisticamente positiva ( $P$-valor $<0.00$ I) com a insuficiência no desenvolvimento de AF. A Tabela 5 apresenta o resultado da regressão linear realizada para analisar a relação entre o sedentarismo e as variáveis observadas neste estudo.

Entre as variáveis observadas, houve correlação significativa somente entre os sexos, não contendo relação entre as demais.

\section{DISCUSSÃO}

Este estudo apresentou a prevalência de sedentarismo em $58,8 \%$ dos adultos sobrepesados e obesos. Identificou como comportamentos sedentários mais comuns nesta população o uso de computadores e televisores e demonstrou correlação positiva entre o sexo feminino e o sedentarismo nesta população.

A maior parte da população do estudo classificada como sedentária não era da raça branca. Estudos realizados com adultos, demonstraram que indivíduos afro-descendentes estão mais propensos a terem níveis baixos de $\mathrm{AF}^{13}$, enquanto os indivíduos brancos são mais propensos a serem suficientemente ativos ${ }^{14}$.-

O sedentarismo prevaleceu nos indivíduos do sexo feminino $65 \%$. Este achado corrobora com um estudo que demonstrou que os homens foram mais ativos que as mulheres no lazer, ao passo que as mulheres relataram maior tempo assistindo à televisão ${ }^{15}$.

Os indivíduos casados representaram a maior parcela de insuficiência de AF e sedentarismo $69 \%$ divergindo de um estudo que demonstrou que adultos solteiros têm maiores chances de desenvolverem comportamentos sedentários, especialmente o uso excessivo do computador ${ }^{16}$. 
TABELA 1 - Características sociodemográficas em relação a atividade física em indivíduos sobrepesados e obesos, Paranavaí PR, Brasil, 2019.

\begin{tabular}{|c|c|c|c|c|c|c|c|c|c|c|}
\hline & M.A & $\%$ & Ativo & $\%$ & I.A & $\%$ & s. & $\%$ & Total & $\%$ \\
\hline \multicolumn{11}{|l|}{ Raça/cor } \\
\hline Branco & 4 & $27 \%$ & 3 & $20 \%$ & 6 & $40 \%$ & 2 & $13 \%$ & 15 & $44 \%$ \\
\hline Não-branco & 2 & $10 \%$ & 5 & $26 \%$ & 7 & $37 \%$ & 5 & $26 \%$ & 19 & $56 \%$ \\
\hline \multicolumn{11}{|l|}{ Sexo } \\
\hline Masculino & 2 & $18 \%$ & 4 & $36 \%$ & 3 & $27 \%$ & 2 & $18 \%$ & 11 & $32 \%$ \\
\hline Feminino & 4 & $17 \%$ & 4 & $17 \%$ & 10 & $43 \%$ & 5 & $22 \%$ & 23 & $68 \%$ \\
\hline \multicolumn{11}{|l|}{ Estado civil } \\
\hline Casados & 4 & $17 \%$ & 3 & $13 \%$ & 9 & $39 \%$ & 7 & $30 \%$ & 23 & $68 \%$ \\
\hline Solteiros & 2 & $18 \%$ & 5 & $45 \%$ & 4 & $36 \%$ & 0 & $0 \%$ & 11 & $32 \%$ \\
\hline \multicolumn{11}{|l|}{ Escolaridade } \\
\hline$\leq 8$ anos de estudo & 0 & $0 \%$ & 2 & $17 \%$ & 7 & $58 \%$ & 3 & $25 \%$ & 12 & $35 \%$ \\
\hline$>8$ anos de estudo & 6 & $27 \%$ & 6 & $27 \%$ & 6 & $27 \%$ & 4 & $18 \%$ & 22 & $65 \%$ \\
\hline \multicolumn{11}{|l|}{ Trabalha } \\
\hline Sim & 5 & $22 \%$ & 6 & $26 \%$ & 9 & $39 \%$ & 3 & $13 \%$ & 23 & $68 \%$ \\
\hline Não & 1 & $9 \%$ & 2 & $18 \%$ & 4 & $36 \%$ & 4 & $36 \%$ & 11 & $32 \%$ \\
\hline \multicolumn{11}{|l|}{ Renda familiar } \\
\hline$\leq 3$ salários mínimos & 4 & $14 \%$ & 8 & $27 \%$ & 10 & $34 \%$ & 7 & $24 \%$ & 29 & $85 \%$ \\
\hline > 3 salários mínimos & 2 & $40 \%$ & 0 & $0 \%$ & 3 & $60 \%$ & 0 & $0 \%$ & 5 & $15 \%$ \\
\hline
\end{tabular}

*MA= Muito Ativos, IA= Irregularmente Ativos, $S=$ Sedentários.

TABELA 2 - Classificação do nível de atividade física de acordo com o IMC. Paranavaí-PR, Brasil.

\begin{tabular}{l|c|c|c|c|c}
\hline Classificação & Sobrepeso/n & $\%$ & Obesidade/n & $\%$ & Total \\
\hline Muito ativo & 2 & $12 \%$ & 4 & $23 \%$ & 6 \\
\hline Ativo & 5 & $29 \%$ & 3 & $18 \%$ & 3 \\
\hline Irregularmente ativo & 7 & $41 \%$ & 6 & $23 \%$ & 13 \\
\hline Sedentário & 3 & $18 \%$ & 4 & $50 \%$ & 7 \\
\hline Total & 17 & $50 \%$ & 17 & 34 \\
\hline
\end{tabular}

TABELA 3 - Prevalência de comportamentos sedentários entre os participantes, Paranavaí PR, Brasil, 2019.

\begin{tabular}{l|c|c|c|c|c|}
\hline \multicolumn{1}{|c|}{ Comportamento Sedentário } & Sobrepesados/n & $\%$ & Obesos/n & $\%$ & Total \\
\hline Televisão & 12 & $70 \%$ & 15 & $88 \%$ & 27 \\
\hline Celular & 13 & $76 \%$ & 15 & $88 \%$ & 28 \\
\hline Computador & 2 & $12 \%$ & 1 & $6 \%$ & 3 \\
\hline Livro & 0 & $0 \%$ & 2 & $12 \%$ & 2 \\
\hline
\end{tabular}

Em relação à escolaridade dos participantes, indivíduos com menos de 8 anos de estudo apresentaram maior prevalência de AF irregular e sedentarismo $83 \%$. Em contrapartida, os indivíduos com maior nível de escolaridade eram propensos a serem mais ativos suficientemente ${ }^{14}$.

A prevalência maior de sedentarismo ocorreu em indivíduos que não trabalham $72 \%$. Este fato faz com que este público-alvo fique mais suscetível a doenças cardiometabólicas, devido a utilização do seu tempo de lazer para prática de comportamentos sedentários ${ }^{17}$. Os participantes com renda familiar $\geq 3$ salários mínimos tiveram maior prevalência na classificação de irregularmente ativos $60 \%$, já aqueles com renda familiar $\leq 3$ salários mínimos apresentaram maior prevalência de sedentarismo $24 \%$.

Relacionado ao IMC, a maioria dos participantes foram classificados como irregularmente ativos, correspondendo a $35 \%$ dos obesos e $41 \%$ dos sobrepesados. Os indivíduos com obesidade apresentaram maior prevalência de sedentarismo $23,5 \%$, tratando-se de um alerta para esta população, visto que demasiados níveis de sedentarismo apresentam efeitos prejudiciais acerca dos fatores de risco cardiovasculares e metabólicos associados a obesidade ${ }^{18}$. Deste modo, os indivíduos acima do peso estão mais propensos a terem menores níveis de $\mathrm{AF}^{13}$.

Existe a possibilidade de que um componente neuro- 
TABELA 4. Consumo alimentar dos indivíduos relacionados a atividade física, Paranavaí-PR, Brasil, 2019.

\begin{tabular}{|c|c|c|c|c|c|c|c|c|c|c|}
\hline Consumo Alimentar & M.A & $\%$ & Ativo & $\%$ & I.A & $\%$ & $\mathbf{s}$ & $\%$ & Total & $\%$ \\
\hline \multicolumn{11}{|l|}{ Frutas } \\
\hline Nunca & 0 & $0 \%$ & 0 & $0 \%$ & 1 & $100 \%$ & 0 & $0 \%$ & 1 & $3 \%$ \\
\hline As vezes & 0 & $0 \%$ & 5 & $31 \%$ & 6 & $37 \%$ & 5 & $31 \%$ & 16 & $47 \%$ \\
\hline Regularmente & 6 & $35 \%$ & 3 & $18 \%$ & 6 & $35 \%$ & 2 & $12 \%$ & 17 & $50 \%$ \\
\hline \multicolumn{11}{|l|}{ Legumes e Verduras } \\
\hline Nunca & 0 & $0 \%$ & 0 & $0 \%$ & 0 & $0 \%$ & 0 & $0 \%$ & 0 & $0 \%$ \\
\hline As vezes & 0 & $0 \%$ & 2 & $33 \%$ & 3 & $50 \%$ & 1 & $17 \%$ & 6 & $18 \%$ \\
\hline Regularmente & 6 & $21 \%$ & 6 & $21 \%$ & 10 & $36 \%$ & 6 & $21 \%$ & 28 & $82 \%$ \\
\hline \multicolumn{11}{|l|}{ Carne vermelha } \\
\hline Nunca & 0 & $0 \%$ & 0 & $0 \%$ & 0 & $0 \%$ & 0 & $0 \%$ & 0 & $0 \%$ \\
\hline As vezes & 0 & $0 \%$ & 1 & $17 \%$ & 4 & $67 \%$ & 1 & $17 \%$ & 6 & $18 \%$ \\
\hline Regularmente & 6 & $21 \%$ & 7 & $25 \%$ & 9 & $32 \%$ & 6 & $21 \%$ & 28 & $82 \%$ \\
\hline \multicolumn{11}{|l|}{ Carne branca } \\
\hline Nunca & 0 & $0 \%$ & 1 & $100 \%$ & 0 & $0 \%$ & 0 & $0 \%$ & 1 & $3 \%$ \\
\hline As vezes & 3 & $18 \%$ & 4 & $23 \%$ & 8 & $47 \%$ & 2 & $12 \%$ & 17 & $50 \%$ \\
\hline Regularmente & 3 & $19 \%$ & 3 & $19 \%$ & 5 & $31 \%$ & 5 & $31 \%$ & 16 & $47 \%$ \\
\hline \multicolumn{11}{|l|}{ Consumo de gordura } \\
\hline Nunca & 1 & $33 \%$ & 0 & $0 \%$ & 1 & $33 \%$ & 1 & $33 \%$ & 3 & $9 \%$ \\
\hline As vezes & 3 & $25 \%$ & 4 & $33 \%$ & 3 & $25 \%$ & 2 & $17 \%$ & 12 & $35 \%$ \\
\hline Regularmente & 2 & $10 \%$ & 4 & $21 \%$ & 8 & $42 \%$ & 4 & $21 \%$ & 19 & $56 \%$ \\
\hline \multicolumn{11}{|l|}{ Consumo de doces } \\
\hline Nunca & 1 & $17 \%$ & 1 & $17 \%$ & 4 & $67 \%$ & 0 & $0 \%$ & 6 & $18 \%$ \\
\hline As vezes & 5 & $24 \%$ & 5 & $24 \%$ & 6 & $28 \%$ & 5 & $24 \%$ & 21 & $62 \%$ \\
\hline Regularmente & 0 & $0 \%$ & 2 & $28 \%$ & 3 & $43 \%$ & 2 & $28 \%$ & 7 & $20 \%$ \\
\hline
\end{tabular}

${ }^{*} \mathrm{MA}=$ Muito Ativos, $\mathrm{IA}=$ Irregularmente Ativos, $\mathrm{S}=$ Sedentários.

TABELA 5 - Análise de correlação entre a atividade física e as variáveis observadas, Paranavaí PR, Brasil, 2019.

\begin{tabular}{|c|c|c|}
\hline Variável & Erro Amostral & p-valor \\
\hline Idade & 1.80 & 0.90 \\
\hline Cor/Raça & 1.78 & 0.47 \\
\hline Sexo & 20156046.08 & 0.00 \\
\hline Estado Civil & 1.82 & 0.44 \\
\hline Nível De Escolaridade & 1.65 & 0.78 \\
\hline Trabalha & 1.43 & 0.86 \\
\hline Renda Familiar & 2.09 & 0.97 \\
\hline IMC & 1.83 & 0.80 \\
\hline Obesidade Abdominal & 2.47 & 0.53 \\
\hline Consumo de Carne Branca & 1.13 & 0.57 \\
\hline Consumo de Carnes Vermelhas & 1.85 & 0.80 \\
\hline Consumo de Doces & 1.15 & 0.74 \\
\hline Consumo de Frutas & 1.42 & 0.75 \\
\hline Consumo de Gordura Vegetal/Animal & 1.09 & 0.82 \\
\hline Consumo de Legumes/Verduras & 1.92 & 0.95 \\
\hline Considera Sua Alimentação Saudável? & 1.38 & 0.92 \\
\hline Consumo de Bebida Alcóolica & 1.63 & 0.83 \\
\hline Tabagismo & 2.10 & 0.66 \\
\hline
\end{tabular}


gênico relacionado ao sedentarismo possa desencadear atividades metabólicas desfavoráveis a saúde e até mesmo ocasionar a hiperfagia no indivíduo, mostrando que o comportamento sedentário está além de ser apenas insuficiência de $\mathrm{AF}^{19}$.

Nesta perspectiva, vale ressaltar que a cada hora extra de sedentarismo pode-se aumentar cerca de $22 \%$ as chances de desenvolver diabetes mellitus tipo 2 e $39 \%$ síndrome metabólica, indiferentemente da prática de $\mathrm{AF}$ de alta intensidade. Portanto, as recomendações destinam-se em reduzir o tempo gasto com atividades de baixa energia, como por exemplo, aquelas executadas na posição sentada ${ }^{16}$.

Neste sentido, ao observar os comportamentos sedentários mais comuns entre os participantes, observouse o uso do celular $82 \%$ e assistir televisão $79 \%$. Corroborando com estudo realizado com 53.034 pessoas, o hábito de assistir à televisão por $\geq 3$ horas diárias foi de $24 \%$, tendo seu aumento gradativo de acordo com a idade e diminuindo com o aumento da escolaridade ${ }^{7}$.

Em uma época onde a modernidade acomoda as pessoas nos seus locais de trabalho e estudo, surge a necessidade do desenvolvimento de estratégias para aumentar a AF nos ambientes relacionados a atuação com computadores e desktops ${ }^{19}$, uma vez que, o sedentarismo tendo ligação direta com o ganho de peso nos indivíduos insuficientemente ativos, torna-se necessário a diminuição do tempo gasto com atividades sedentárias ${ }^{14}$.

Dentre os participantes que relataram fazer consumo de bebida alcoólica $41 \%$, foram classificados como irregularmente ativos e dois como sedentários. Um estudo relatou que indivíduos com maior ingestão de bebida alcoólica são mais propensos a serem suficientemente ativos ${ }^{14}$.

Neste estudo o consumo de gorduras e doces foi comum entre os participantes classificados com AF insuficiente ou sedentarismo, além de que estes indivíduos consideraram, em sua maioria, ter uma alimentação nãosaudável. Comportamentos sedentários simultâneos que envolvem atividades com um gasto energético muito baixo (I,0-I,8 equivalentes metabólicos), realizados principalmente em posição sentada ou em decúbito dorsal, foram associados ao consumo de alimentos não saudáveis em adultos ${ }^{20}$.

Diante dos desafios encontrados na adoção de hábitos saudáveis pela população, os profissionais de enfermagem, dentro de suas diretrizes, devem aprimorar seus conhecimentos acerca das práticas relacionadas a um estilo de vida saudável na população obesa e sobrepesada, utilizando da promoção de intervenções de saúde em larga escala, a fim de reduzir o tempo gasto com comportamentos sedentários ${ }^{21}$.

Um estudo demonstrou que o aconselhamento na atenção primária acerca do comportamento sedentário representa apenas $10 \%$ dos correlatos dos pacientes, sendo que, somente $2 \%$ relatam um aconselhamento detalhado sobre seu comportamento sedentário, demonstrando baixa frequência no debate desse assunto pelos profissionais da saúde com seus pacientes. Diante do exposto, deve-se estabelecer uma base de informações para o planejamento de intervenções comportamentais sedentárias a ser implantado nos indivíduos, avaliando posteriormente a efetividade do programa no ambiente clínico ${ }^{22}$.

Um ensaio clínico randomizado demonstrou que intervenções no estilo de vida sob medida realizadas no domicílio, desenvolvidas pela atenção primária à saúde por meio de reuniões presenciais, telefonemas de aconselhamentos, materiais educativos impressos e em DVDs sobre AF e perda de peso, com foco na promoção da perda de peso e no aumento da AF, foram eficazes no alcance de seus objetivos ${ }^{23}$.

Como limitações deste estudo apontam-se a amostra pequena, restrita a uma única UBS por se tratar de um estudo piloto. Esta amostra dificulta a generalização dos dados apresentados, porém serviu como indicador de melhoria no processo de coleta de dados para a etapa do estudo principal, demonstrando a necessidade de realizar uma melhor divulgação do estudo com a população e profissionais de saúde antes de iniciar a coleta de dados. Pretende-se em estudo posterior realizar a coleta de dados com uma amostra mais abrangente.

\section{CONCLUSÃO}

A maioria da população obesa e sobrepesada apresentou comportamento sedentário neste estudo. $O$ comportamento sedentário é fator de risco para o aumento de peso principalmente quando aliado ao consumo de alimentos não saudáveis. Este estudo demonstrou que dentre esta população o comportamento sedentário esteve mais frequente entre as mulheres.

Neste sentido as ações de estímulo à prática de $\mathrm{AF}$ regular devem ser ampliadas entre as mulheres, com sobrepeso e obesidade.As informações obtidas neste estudo devem contribuir para a elaboração de estratégias de prevenção e promoção da saúde direcionadas ao público com sobrepeso e obesidade. Promovendo ao longo das ações educativas, os pontos favoráveis na busca de um estilo de vida saudável e enfrentando as dificuldades para a adoção de hábitos saudáveis pelos indivíduos. 


\section{REFERÊNCIAS}

I. González K, Fuentes J, Márquez JL. Physical Inactivity, Sedentary Behavior and Chronic Diseases. Korean J Fam Med 2017; 38: III-II5.

2. Tremblay MS, Aubert S, Barnes JD, Saunders TJ, Carson V, Latimer-Cheung AE et al. Rede de Pesquisa de Comportamento Sedentário (SBRN) - processo e resultados do Projeto de Consenso de Terminologia. 10/06/2017. 2017. Disponível em: https://www.sedentarybehaviour.org/sbrnterminology-consensus-project/portuguese-brazil-translation/ (acessado 3 maio 2019).

3. WHO /What is Moderate-intensity and Vigorous-intensity Physical Activity? WHO 2014.

4. Aziz JL. Sedentarismo e Hipertensão Arterial. Rev Bras Hipertens 20I4; 2I: 75-82.

5. Andriolo V, Dietrich S, Knüppel S, Bernigau W, Boeing $\mathrm{H}$. Traditional risk factors for essential hypertension: analysis of their specific combinations in the EPIC-Potsdam cohort. Sci Rep. 2019; 9: I50I.

6. World Health Organization. Physical activity. 2018. Disponível em: https:/www.who.int/en/news-room/fact-sheets/ detail/physical-activity (acessado 3 maio 2019).

7. Brasil. Ministério da Saúde. Secretaria de Vigilância em Saúde. Departamento de Análise em Saúde e Vigilância de Doenças não Transmissíveis.Vigitel Brasil 2018: vigilância de fatores de risco e proteção para doenças crônicas por inquérito telefônico: estimativas sobre frequência e distribuição sociodemográfica de fatores de risco e proteção para doenças crônicas nas capitais dos 26 estados brasileiros e no Distrito Federal em 2018. Brasília: Ministério da Saúde; 2019.

8. Brasil, Ministério da Saúde. Sobre a Vigilância de DCNT. 2019. Disponível em: http://portalms.saude.gov.br/noticias/43036-sobre-a-vigilancia-de-dent (acessado 3 maio 2019).

9. World Health Organization. Obesity and overweight. 2018.Disponível em: https:/www.who.int/en/news-room/fact-sheets/ detail/obesity-and-overweight (acessado 3 maio 2019).

I0. IDF. International Diabetes Foundation. Consensus Worldwide definition of the Metabolic Synfrome. 2006. Disponível em: https://www.idf.org/e-library/consensus-statements/60-idfconsensus-worldwide-definitionof-the-metabolic -syndrome (acessado 7 maio 2019).

I I. Instituto Brasileiro de Geografia e Estatística. Ministério do Planejamento, Orçamento e Gestão. Coordenação de Trabalho e Rendimento. PNS 2013: Manual de antropometria. Disponível em: https://www.pns.icict.fiocruz.br/arquivos/ Novos/Manual de Antropometria PDF.pdf. (acessado 17 out 2019).

12. Renato Pardini, Sandra Matsudo T, Araújo, Victor Matsudo EA, Glaucia Braggion, Douglas Andrade L, Oliveira AFJ e VR.
Validation of the International Physical Activity Questionaire (IPAQ version 6): pilot study in Brazilian young adults. Rev Bras Ciência e Mov. 200I; 9: 318.

13. Unick JL, Lang W, Tate DF, Bond DS, Espeland MA, Wing RR. Objective Estimates of Physical Activity and Sedentary Time among Young Adults. J Obes 2017; 2017: 9257564.

14. Gibbs BB, Gabriel KP, Carnethon MR, Gary-Webb T, Jakicic JM, Rana JS et al. Sedentary Time, Physical Activity, and Adiposity: Cross-sectional and Longitudinal Associations in CARDIA.Am J Prev Med 2017; 53: 764.

15. Mielke GI, Hallal PC, Rodrigues GBA, Szwarcwald CL, Santos FV, Malta DC. Prática de atividade física e hábito de assistir à televisão entre adultos no Brasil: Pesquisa Nacional de Saúde 2013. Epidemiol e Serviços Saúde 2015; 24 : 277-286.

16. Huffman S, Szafron M. Social correlates of leisure-time sedentary behaviours in Canadian adults. Prev Med Reports 20I7; 5: 268-274.

17. Lim MS, Park B, Kong IG, Sim S, Kim SY, Kim J-H et al. Leisure sedentary time is differentially associated with hypertension, diabetes mellitus, and hyperlipidemia depending on occupation. BMC Public Health 2017; 17: 278.

18. Leiva AM, Martínez MA, Cristi-Montero C, Salas C, Ramírez-Campillo $R$, Díaz Martínez $X$ et al. El sedentarismo se asocia a un incremento de factores de riesgo cardiovascular y metabólicos independiente de los niveles de actividad física. Rev Med Chil 2017; I45: 458-467.

19. Panahi S, Tremblay A. Sedentariness and Health: Is Sedentary Behavior More Than Just Physical Inactivity? Front public Heal 2018; 6: 258.

20. Compernolle S, De Cocker K, Teixeira PJ, Oppert J-M, Roda C, Mackenbach JD et al. The associations between domain -specific sedentary behaviours and dietary habits in European adults: a cross-sectional analysis of the SPOTLIGHT survey. BMC Public Health 2016; 16: 1057.

21. Kable A, James C, Snodgrass S, Plotnikoff R, Guest M, Ashby $S$ et al. Nurse provision of healthy lifestyle advice to people who are overweight or obese. Nurs Health Sci 2015; 17: 45I-459.

22. Shuval K, DiPietro L, Skinner CS, Barlow CE, Morrow J, Goldsteen R et al. "Sedentary behaviour counselling": the next step in lifestyle counselling in primary care; pilot findings from the Rapid Assessment Disuse Index (RADI) study. Br J Sports Med 20।4; 48: |45|-5.

23. Eaton CB, Hartman SJ, Perzanowski E, Pan G, Roberts MB, Risica PM et al. A Randomized Clinical Trial of a Tailored Lifestyle Intervention for Obese, Sedentary, Primary Care Patients. Ann Fam Med 2016; 14: 31 I-9.

Recebido: 2020-04- 12

Aceito: 2020-05-12 